\title{
Small maritime target detection through false color fusion
}

\author{
Alexander Toet ${ }^{1 \mathrm{a}}$, Tirui $\mathrm{Wu}^{2 \mathrm{~b}}$ \\ ${ }^{a}$ TNO Human Factors, P.O. Box 23, 3769 ZG Soesterberg, the Netherlands \\ b Jiangsu Univ. of Science and Technology, P. O. Box 326, Zhenjiang Jiangsu 212003, P. R. China
}

\begin{abstract}
We present an algorithm that produces a fused false color representation of a combined multiband IR and visual imaging system for maritime applications. Multispectral IR imaging techniques are increasingly deployed in maritime operations, to detect floating mines or to find small dinghies and swimmers during search and rescue operations. However, maritime backgrounds usually contain a large amount of clutter that severely hampers the detection of small targets. Our new algorithm deploys the correlation between the target signatures in two different IR frequency bands (3-5 and 8-12 $\mu \mathrm{m})$ to construct a fused IR image with a reduced amount of clutter. The fused IR image is then combined with a visual image in a false color RGB representation for display to a human operator. The algorithm works as follows. First, both individual IR bands are filtered with a morphological opening top-hat transform to extract small details. Second, a common image is extracted from the two filtered IR bands, and assigned to the red channel of an RGB image. Regions of interest that appear in both IR bands remain in this common image, while most uncorrelated noise details are filtered out. Third, the visual band is assigned to the green channel and, after multiplication with a constant (typically 1.6) also to the blue channel. Fourth, the brightness and colors of this intermediate false color image are renormalized by adjusting its first order statistics to those of a representative reference scene. The result of these four steps is a fused color image, with naturalistic colors (bluish sky and grayish water), in which small targets are clearly visible.
\end{abstract}

Keywords: target detection; false color fusion, small targets, clutter, image fusion

\section{INTRODUCTION}

In this paper, we discuss the problem of small low observable (dim) target detection in cluttered maritime infrared images. This problem is of interest in many maritime operations, such as the detection of floating mines or finding dinghies and swimmers during search and rescue operations.

In the literature there are many efficient algorithms for the detection and tracking of targets of significant size. However, maritime backgrounds usually contain a large amount of clutter that severely hampers the detection of small targets. Clutter may have many causes. Sea surface structure, reflection and emission changes related to incident angle variations and surface effects are standard features governing clutter behavior ${ }^{3}$. Sun glint and horizon effects also contribute to clutter. The existence of scanline disturbance and noise further increases the difficulty in proper detection.

Here we present a new algorithm that produces a fused false color representation of a combined multiband IR and visual imaging system. The method efficiently maps a combination of infrared and visual imagery into RGB space and yields a natural looking representation of the inspected scene. This representation makes targets more detectable, which will evidently result in improved detection performance. The rest of this paper is organized as follows. In Section 2, a brief introduction to morphological operations is given because our new target detection is based on a morphological filter: the top-hat transform. We introduce our new method in detail in Section 3. In Section 4, some experimental results are shown. Finally, the conclusions are drawn in Section 5.

\footnotetext{
${ }^{1}$ lex.toet@tno.nl; phone +31-346-356237; fax +31-346-353977; http://lextoet.googlepages.com/

2 tirui_wu@163.com; Jiangsu Univ. of Science and Technology, P. O. Box 326, Zhenjiang Jiangsu 212003, P. R. China
} 


\section{MORPHOLOGICAL OPERATIONS}

Morphological operations are powerful non-linear filters, and they are widely used in image processing, for feature extraction, image enhancement, target detection, etc ${ }^{1}$. In this section we briefly define the basic morphological transformations for discrete (e.g. sampled) functions that are used in our new image fusion scheme. For an extensive introduction to mathematical morphology we refer to the literature ${ }^{1,4}$.

Two basic operations, dilation and erosion, are fundamental to morphological processing. Dilation is defined as:

$$
A \oplus B=\left\{z \mid(\hat{B})_{z} \bigcap A \neq \varnothing\right\}
$$

where $A$ and $B$ are sets in $Z^{2}$ (integer space), $A$ is dilated by $B$. This equation is based on obtaining the reflection of $\mathrm{B}$ about its origin and shifting this reflection by $z$. The dilation of $A$ by $B$ then is the set of all displacements, $z$, such that $\hat{B}$ and $A$ overlap by at least one element

Erosion is defined as:

$$
A \odot B=\left\{z \mid(\hat{B})_{z} \subseteq A\right\}
$$

Hence, the erosion of $A$ by $B$ is the set of all points $z$ such that $B$, translated by $z$, is contained in $A$.

The combination of dilation and erosion operations yields two other important morphological operations: opening and closing. The opening $\mathrm{A}$ by $B$ is the erosion of $A$ by $B$, followed by a dilation of the result by $B$. The closing of $A$ by $B$ is the dilation of $A$ by $B$, followed by the erosion of the result by $B$. They are defined by equations 3 and 4 , respectively.

$$
A \circ B=(A \odot B) \oplus B
$$

$$
A \bullet B=(A \oplus B) \odot B
$$

The top-hat operation, which plays a crucial role in our algorithm, can now be defined as ${ }^{2}$ :

$$
h=f-(f \circ b)
$$

where $f$ (special type of $A$ ) is the original image, $b$ is a structuring element (special type of $B$ ), and $h$ is the processed result. The top-hat operation is for instance useful to enhance or extract details in the presence of shading. In most maritime applications, we are only interested in objects which have a temperature which is high relative to the local background (small maritime targets are usually surrounded by water of a lower temperature). In infrared imagery these targets are depicted as bright spots on a darker background. So, we can use the top-hat transform to extract regions from maritime image which contain potential small targets.

\section{METHOD}

In this section, we introduce a new method that produces a fused false color representation of a combined multiband IR and visual imaging system for maritime applications. Our new algorithm deploys the correlation between the target signatures in two different IR frequency bands (3-5 and 8-12 $\mu \mathrm{m})$ to construct a fused IR image with a reduced amount of clutter. The fused IR image is then combined with a visual image in a false color RGB representation for display to a human operator. Fig. 1 shows the scheme of the proposed algorithm. The algorithm works as follows.

First, both individual IR bands are filtered with a morphological opening top-hat transform to extract small details. After performing the top-hat operation, we get two filtered IR bands in which potential targets remain but the background is suppressed. 
Second, a common IR image is extracted from these two filtered IR bands. Regions of interest that appear in both IR bands remain in this common IR image, while most uncorrelated noise details are filtered out. In this step, we use smaller values of those two filtered IR bands to compose the common image. Because regions of interest may have small grayscale value, we may multiply the common IR image with a factor. We adopt a simple method to compute this factor:

$$
\text { factor }=\frac{255}{\operatorname{Sum}\left(\operatorname{Max}_{50}\left(I_{\text {com }}\right)\right) / 50}
$$

where $I_{\text {com }}$ is the common image and $\operatorname{Max}_{50}$ represents the first fifty largest grayscale values of the common IR image.

The common IR image is then assigned to the red channel of an RGB image.

Third, the visual band is assigned to the green channel and, after multiplication with a constant to the blue channel. The constant controls the proportion between green and blue channel: by changing it we can get a different appearance of the fused image. In our experiment, we set the constant equal to 1.6.

Fourth, the brightness and colors of this intermediate false color image are renormalized by adjusting its first order statistics to those of a representative reference scene. Let ini be the initial false color image and ref be the reference image. The three steps of this method are as follows.

(1) Convert both the initial false color image $\left[R_{i n i}, G_{i n i}, B_{i n i}\right]$ and the reference image $\left[R_{r e f}, G_{r e f}, B_{r e f}\right]$ from RGB space into HSI color space. Let $\left[H_{i n i}, S_{i n i}, I_{i n i}\right]$ and $\left[H_{r e f}, S_{r e f}, I_{r e f}\right]$ be the three components of initial false color image and the reference image. The three components in HSI space are independent, so we can adjust one component without changing the other two components.

(2) Match the first order statistics of the value distributions of the saturation and intensity channels between Iini and a color reference image ref using a linear remapping in HSI color space:

$$
\text { result }=\frac{\sigma_{r e f}^{(i)}}{\sigma_{i n i}^{(i)}}\left(C_{i n i}^{(i)}-\mu_{i n i}^{(i)}\right)+\mu_{r e f}^{(i)} \quad ; \quad i=2,3
$$

where $C_{i n i}^{(i)}$ is the $i^{\text {th }}$ channel of initial fused image in HSI space, $\left(\mu_{i n i}^{(i)}, \sigma_{i n i}^{(i)}\right)$ and $\left(\mu_{r e f}^{(i)}, \sigma_{r e f}^{(i)}\right)$ correspond to each channel's mean and standard deviation of the initial fused image and the reference image, respectively.

Then, replace $C_{i n i}^{(i)}$ by result. Note that we don't change channel 1 (the Hue channel). Perform the inverse transformation to obtain the final color fused image.

\section{EXPERIMENT}

We tested our method on two registered multiband image sequences representing a dynamic maritime scene with three kayaks at high sea and approaching from far away. The cameras that were used to register this imagery were respectively:

- $\quad$ a Radiance HS IR camera (Raytheon), sensitive for 3-5 $\mu \mathrm{m}$,

- $\quad$ an AIM $256 \mu \mathrm{LW}$ camera (AEG), sensitive for 8-10 $\mu \mathrm{m}$, and

- $\quad$ a Philips LTC500 CCD camera (f/1.2 50dB s/n at 0.4 Lux) sensitive from visual to near IR (400-900 nm).

Full details of the image registration procedure have been presented elsewhere ${ }^{5}$.

Fig. 2 shows a picture of a beach which was used as the reference image in the experiment. Figs. 3-6 show a set of typical maritime images, representing three kayaks (located approximately in the middle of the scene) at high sea. The first three images of each of these figures are respectively obtained with the Radiance 3-5 $\mu$ m, the AIM 8-12 $\mu \mathrm{m}$ IR and the CCD camera. The last image of each of these figures is the result of our new image fusion scheme. 

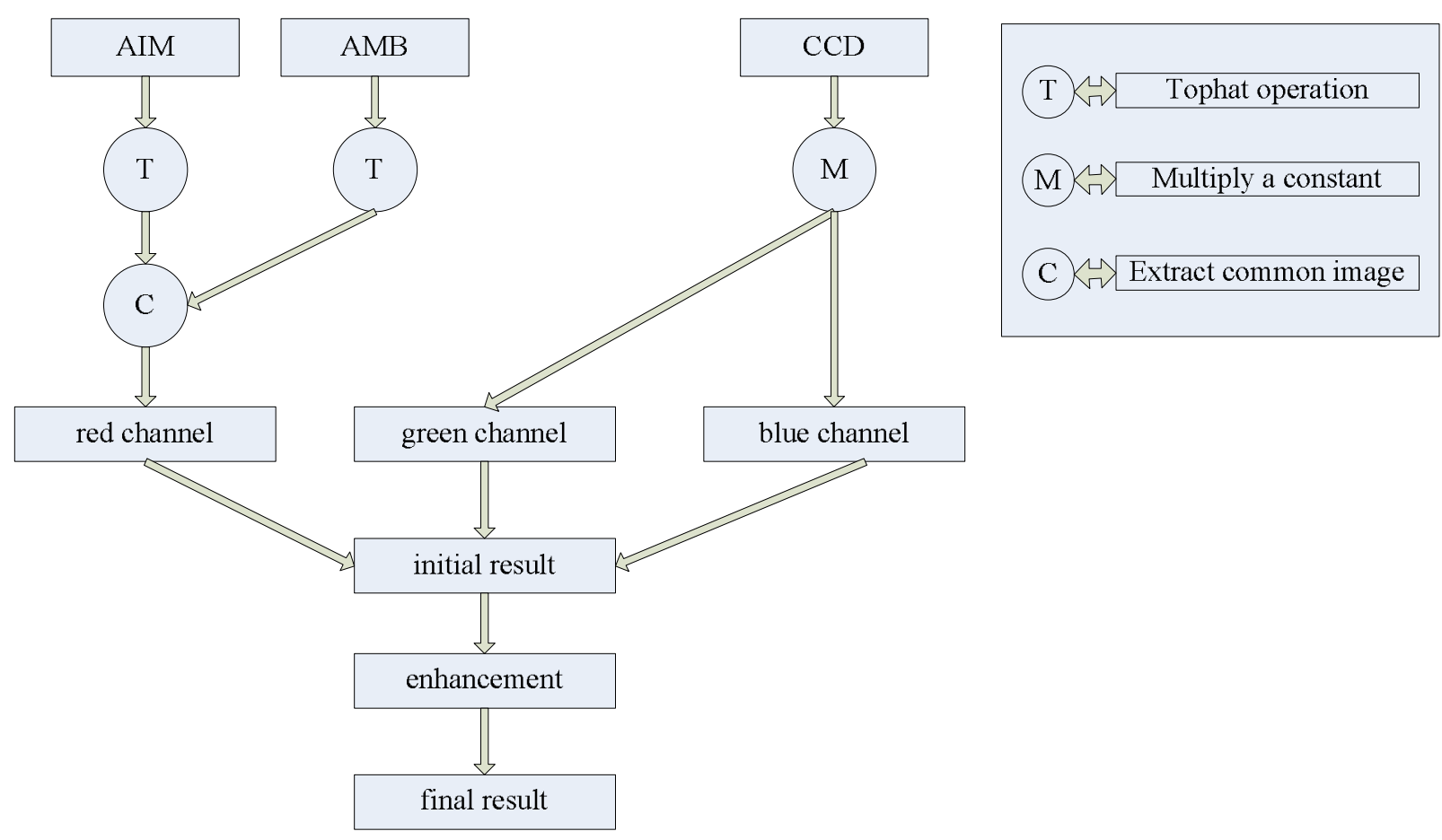

Fig. 1. Scheme of the new image fusion method

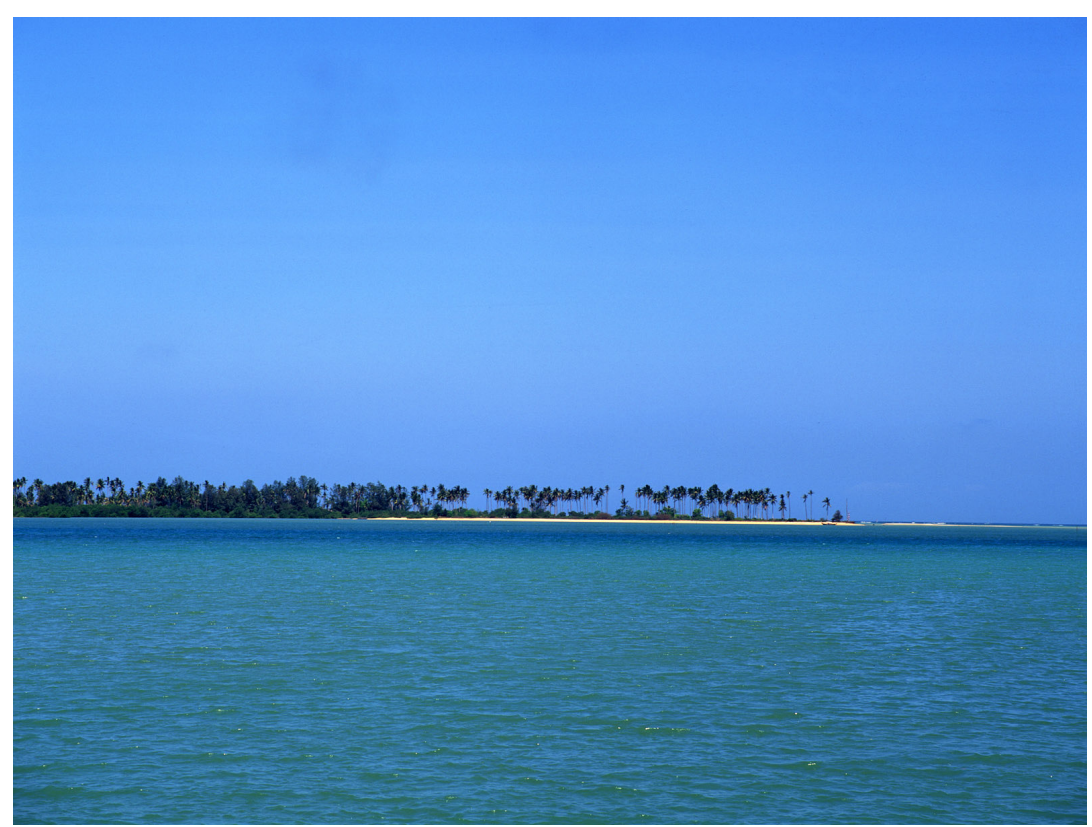

Fig.2. Reference image. 

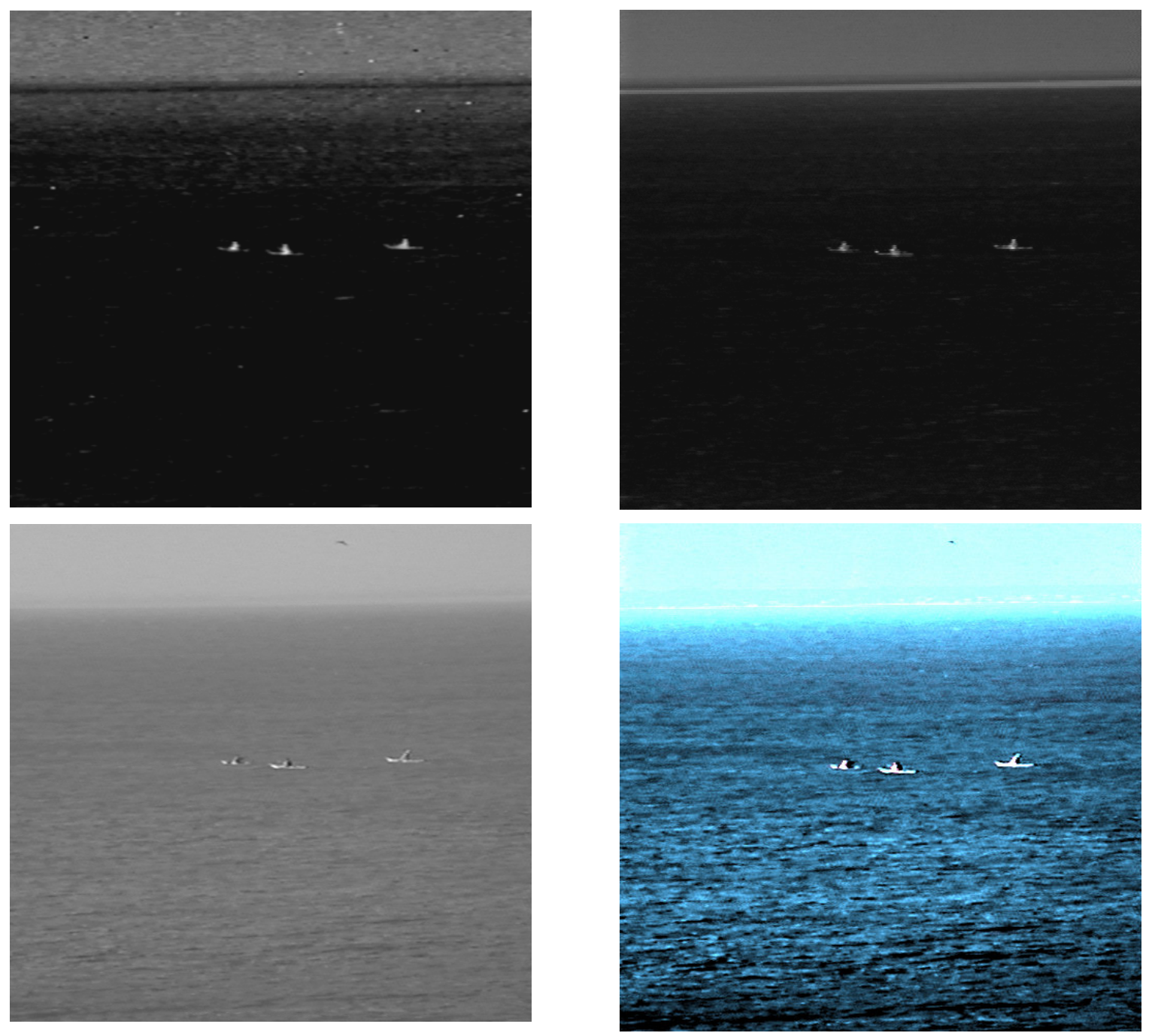

Fig.3. Upper row: corresponding original Radiance 3-5 $\mu \mathrm{m}$ (left) and AIM 8-12 $\mu \mathrm{m}$ (right) IR images. Lower row: corresponding original CCD (left) and fused (right) images. 

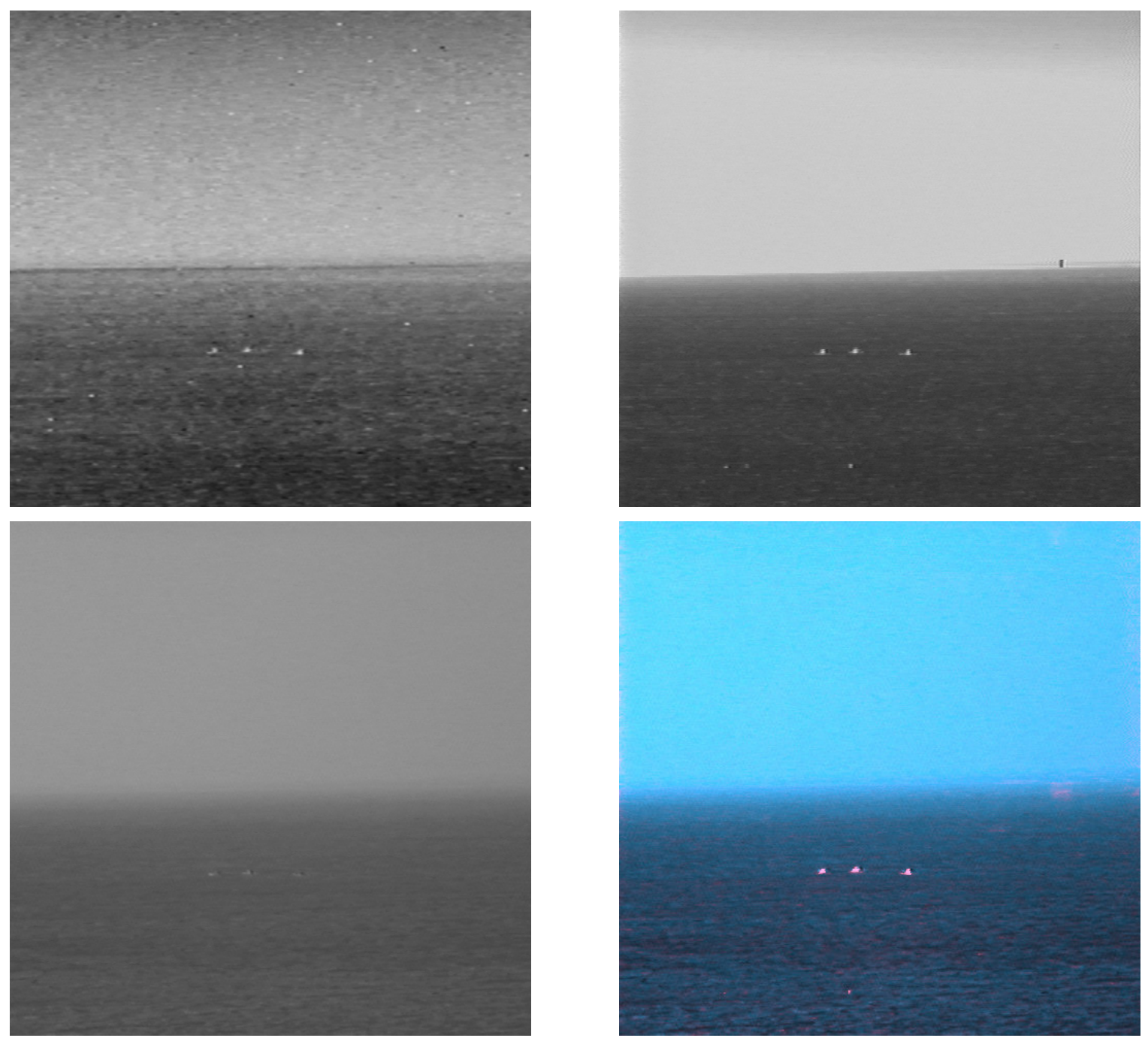

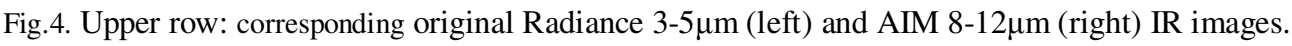
Lower row: corresponding original CCD (left) and fused (right) images. 

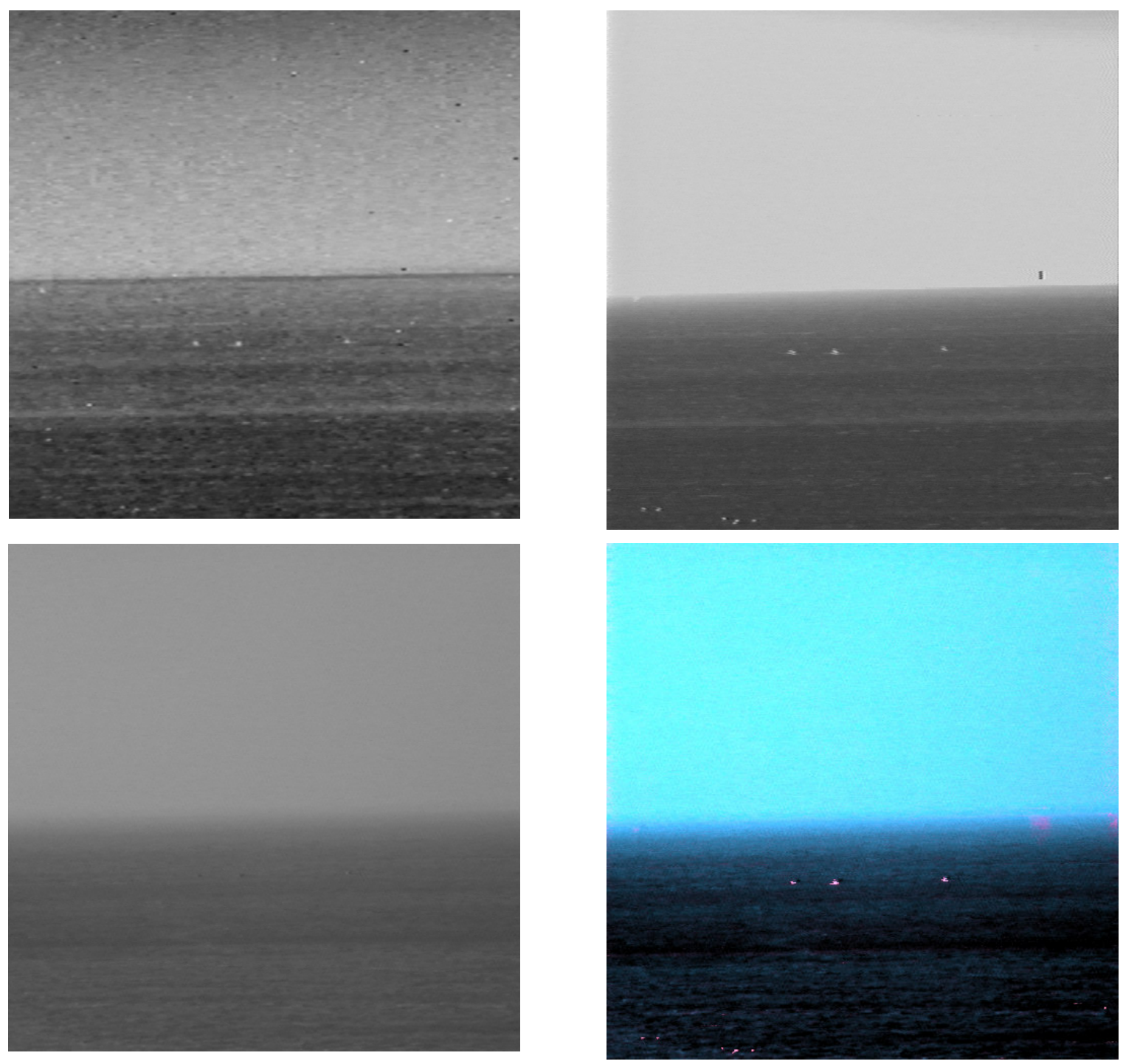

Fig.5. Upper row: corresponding original Radiance 3-5 $\mu \mathrm{m}$ (left) and AIM 8-12 $\mu \mathrm{m}$ (right) IR images. Lower row: corresponding original CCD (left) and fused (right) images. 

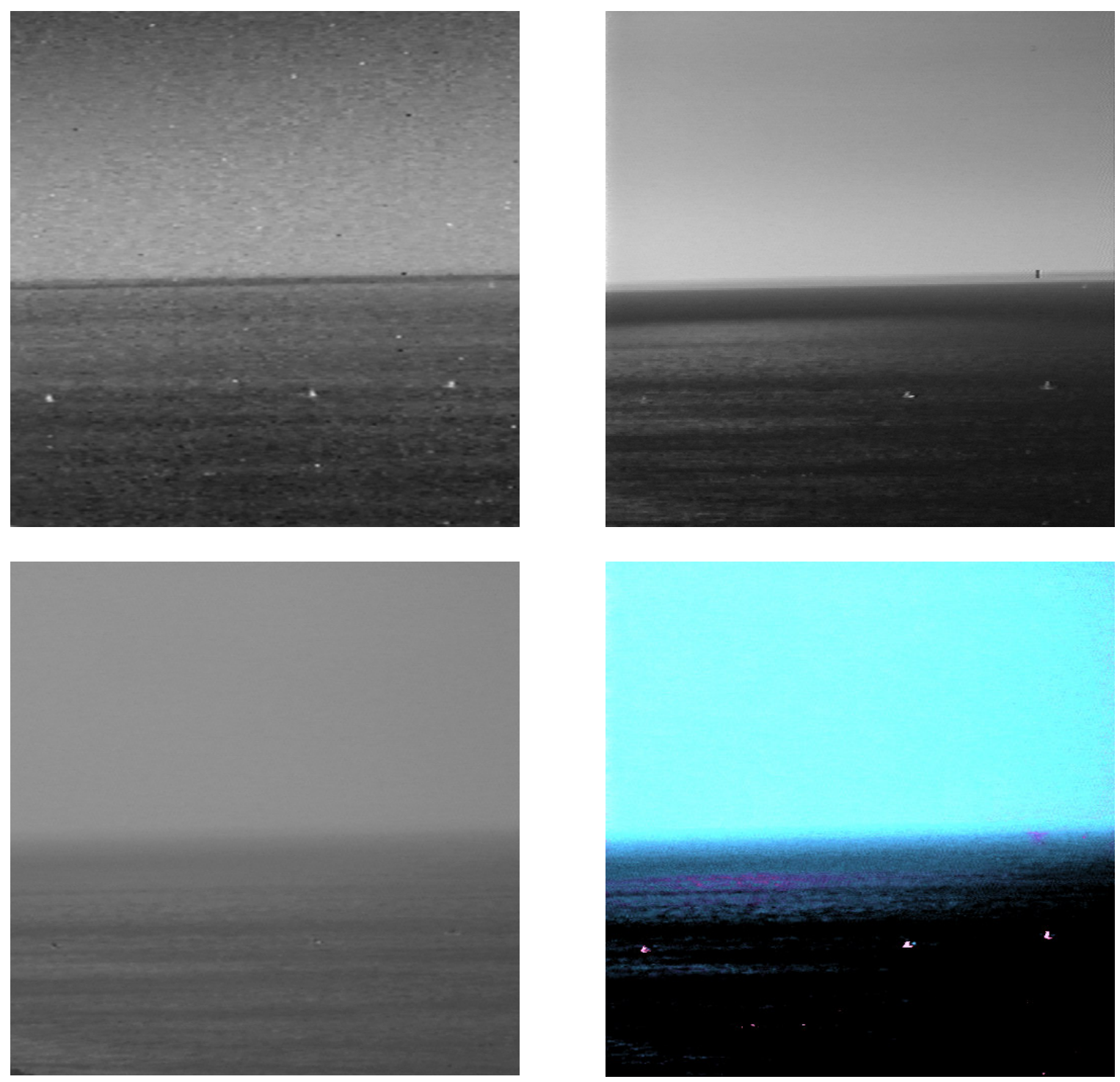

Fig.6. Upper row: corresponding original Radiance 3-5 $\mu \mathrm{m}$ (left) and AIM 8-12 $\mu \mathrm{m}$ (right) IR images. Lower row: corresponding original CCD (left) and fused (right) images.

\section{CONCLUSIONS}

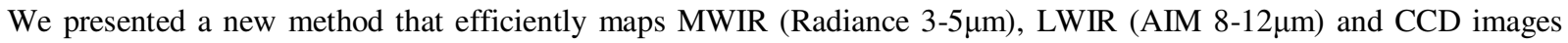
into RGB space, and enhances the representation of small maritime targets. Moreover, this method can easily be implemented in real-time using low-cost hardware, to enhance the detectability of small targets in dynamic conditions However, this method still leaves room for further improvement, such as the selection of a suitable reference image, etc. In our experiments, we found that the reference image determined the appearance of the final fused image. 


\section{REFERENCES}

1. Dougherty, E.R. and Lotufo, R.A., Hands-on morphological image processing, The Society of Photo-Optical Instrumentation Engineers, Bellingham, Washington, 2003.

2. Meyer, F., Contrast feature extraction, In: J.L. Chermant (Ed.), Quantitative analysis of microstructure in material sciences, biology and medicine, pp. Riederer Verlag, Stuttgart, GE, 1978.

3. Schwering, P.B., Maritime infrared background clutter, In: W.R. Watkins \& D. Clement (Ed.), Targets and backgrounds: characterization and representation II, pp. 255-266, The International Society for Optical Engineering, Bellingham,WA, 1996.

4. Serra, J., Image analysis and mathematical morphology, Academic Press, London, UK, 1982.

5. Toet, A., Detection of dim point targets in cluttered maritime backgrounds through multisensor image fusion, In: W.R. Watkins, D. Clement \& W.R. Reynolds (Ed.), Targets and Backgrounds: Characterization and Representation VIII, pp. 118-129, The International Society for Optical Engineering, Bellingham, WA, 2002. 